\title{
Ultrastructure of Sarcocystis bertrami sarcocysts from a naturally infected donkey (Equus asinus) from Egypt
}

\author{
J. P. DUBEY ${ }^{1, *}$, E. VAN WILPE ${ }^{2}$, S. K. VERMA ${ }^{1}$, M. HILALI ${ }^{3}$, \\ ${ }^{I}$ U. S. Department of Agriculture, Agricultural Research Service, Beltsville Agricultural Research Center, Animal \\ Parasitic Diseases Laboratory, Building 1001, Beltsville, MD 20705-2350, USA \\ ${ }^{2}$ Department of Anatomy and Physiology, Faculty of Veterinary Science, University of Pretoria, Onderstepoort, \\ 0110, South Africa \\ ${ }^{3}$ Parasitology Department, Faculty of Veterinary Medicine, Cairo University, 12211, Giza, Egypt
}

Running Head: Sarcocystis bertrami from the donkey

*Corresponding author: USDA, ARS, APDL, BARC-East, Building 1001, Beltsville, MD 20705, USA. Tel. +1 301

504 8128. Fax: +1 301504 9222. E-mail: jitender.dubey@ars.usda.gov

\section{Summary}

There is considerable confusion concerning Sarcocystis species in equids. Little is known of Sarcocystis infections in donkeys (Equus asinus). Here we describe the structure of Sarcocystis bertrami from the donkey by light and transmission electron microscopy (LM, TEM). Nineteen sarcocysts from the tongue of a donkey from Egypt were studied both by LM and TEM. By LM, all sarcocysts had variably shaped and sized projections on the cyst walls, giving it a thin walled to thick walled appearance, depending on individual cyst and plane of section. By TEM, sarcocysts walls had villar protrusions (vp) of type 11. The sarcocyst wall had conical to slender vp, up to $6 \mu \mathrm{m}$ long and $1 \mu \mathrm{m}$ wide; the vp were folded over the cyst wall. The 
total thickness of the sarcocyst wall with ground substance layer (gs) was 1-3 $\mu \mathrm{m}$. The vp had microtubules that originated deeper in the gs and continued up to the tip. The apical part of the vp had electron dense granules. The microtubules (mt) were configured into three types: a tuft of electron dense mt extending the entire length of the vp with a tuft of medium electron dense mt appearing in parallel, and fine mt present only in the villar tips. The gs was mainly smooth with few indistinct granules. The gs was mainly smooth with few indistinct granules. All sarcocysts were mature and contained metrocytes and bradyzoites. Bradyzoites were approximately 11-15 x 2-3 $\mu \mathrm{m}$ in size with typical organelles.

Key words: Sarcocystis bertrami; Donkey (Equus asinus); Transmission electron microscopy

\section{Introduction}

There is considerable confusion concerning the validity of different species of Sarcocystis in the horse and other equids (Dubey et al. 2015). Five species, Sarcocystis bertrami, Sarcocystis neurona, Sarcocystis fayeri, Sarcocystis equicanis, and Sarcocystis asinus have been named (Dubey et al. 2015). Of these, S. neurona is transmitted by Didelphis virginiana and is confined to the Americas, coinciding with the geographic distribution of the opossum. Sarcocystis fayeri, originally described from the USA, has been found in Germany; the domestic dog is its definitive host. Sarcocystis fayeri sarcocysts are thick-walled and has characteristic slender villar protrusions. Sarcocystis bertrami was named by Doflein (1901) in his book. The description was based on earlier observations by others (Siedamgrotzky, 1872, Bertram, 1892 -we do not have access to these papers). Its description is vague and very brief. Rommel and Geisel (1975) found a thin-walled sarcocyst in Germany with dog as the definitive host; they named the parasite, $S$. equicanis. Dubey et al. (2015) reviewed the literature and synonomized S.equicanis with $S$. 
bertrami because the identity of the original $S$. bertrami will remain unknown as there are no archived specimens. Sarcocystis bertrami has not been reported from the Americas. Both $S$. fayeri and S.bertrami/S.equicanis have been reported from Germany (Erber and Geisel, 1981).

Gadaev (1978) named a new species, S. asinus from the donkey, based on a very cryptic morphological description of the sarcocyst. This species was declared species inquirende/invalid by several authors (Levine and Tadros,1980; Odening,1998; Dubey et al. 2015).

Part of the confusion concerning the identity of Sarcocystis species in equids is attributable to inadequate desriptions of the parasite, specially from the donkey.

Donkeys are important for the economy of Africa, Asia, and the Arabian Peninsula, and their popularity is increasing in Europe. Sarcocysts have been found in donkeys from Egypt, Austria, Russia (former USSR), and Great Britain but the parasite has not been adequately described (Gadaev, 1978 ; Hilali and Nasser, 1987 ; Hinaidy and Loupal, 1982 ; Edwards, 1984 ; Kirmse, 1986 ; Zayed and El-Ghaysh, 1998).

The objective of the present paper is to describe the structure of sarcocysts in a donkey.

\section{Materials and methods}

An adult donkey was obtained live on 14 January 2015 from El Fayoum Governorate, Egypt for teaching post mortem technique to veterinary students. The donkey was euthanized by the Pathology Department, Faculty of Veterinary Medicine, Cairo University,Giza, Egypt.

For the present study, tongue tissue was fixed in glutaraldehyde (GF) and formalin. The formalin-fixed (FF) tissue was processed for paraffin embedding. The paraffin blocks and the glutaraldehyde fixed samples were transported to the Faculty of Veterinary Science, University of Pretoria, Onderstepoort, South Africa for light and electron microscopic examinations. For light microscopy (LM), paraffin-embedded sections were cut at $5 \mu \mathrm{m}$ thick and examined after staining with hematoxylin and 
eosin (H and E). For transmission electron microscopy (TEM), glutaraldehyde-fixed tissue was processed using standard techniques. Briefly, the samples wase post-fixed in $1 \%$ osmium tetroxide in Millonig's buffer (pH 7.4), dehydrated through a series of graded ethanols, infiltrated with an epoxy resin/propylene oxide mixture before being embedded in absolute resin, and polymerized at $60^{\circ} \mathrm{C}$ overnight. Two cysts were detected in the glutaraldehyde-fixed sample. A further 17 tissue cysts, located in paraffin blocks (by matching with $\mathrm{H}$ and E sections) were deparaffinised (Van den Berg Weermans and Dingemans, 1984). Ultrathin resin sections were contrasted with uranyl acetate and lead citrate and examined in a Philips CM10 transmission electron microscope (FEI, Eindhoven, The Netherlands) operated at $80 \mathrm{kV}$.

\section{Results}

In total, 19 sarcocysts from 1 donkey were studied ultrastructurally; 2 from glutaraldehyde and 17 from paraffin blocks. Sarcocysts were microscopic, and up to $110 \mu \mathrm{m}$ wide. By light microscopy of $5 \mu \mathrm{m}$ histological sections, the cyst wall thickness varied from $<1$ $\mu \mathrm{m}$ to $5 \mu \mathrm{m}$, depending on the individual cyst and the plane of section (Fig.1). Some cysts had villar protrusions (vp) that varied in shape and size. Examples from 9 sarcocysts are shown in Figure 1.

By TEM of glutaraldehyde sarcocysts, the parasitophorous vacuolar membrane (pvm) was undulating, and lined by an electron dense layer (edl). The edl was thinned out and invaginated giving the pvm a vacuolated appearance (Figs. 2,3). The pvm was folded into villar protrusions (vp) that varied in shape and size, within the same cyst (Fig. 2 B ,C). In some cysts, vp were adjacent to each other, while in others they were $2 \mu \mathrm{m}$ apart. Villar protrusions were mostly elongated, up to $6 \mu \mathrm{m}$ long and up to $1 \mu \mathrm{m}$ wide, with some showing a conical shape. Electron dense, evenly distributed hair-like structures were seen on vp tips, both in glutaraldehyde- and formalin-fixed vp (Fig. 3A). The vp contained microtubules (mt) that varied 
in ultrastructure. A tuft of highly electron dense mt extended from the tip of the villus deep into the interior of the cyst reaching the pellicle of the zoites (Fig. 2C). Another group of less electron dense $\mathrm{mt}$ existed parallel to the electron dense $\mathrm{mt}$ (Fig. 2C). Additionally, fine mt were located towards the tips of the vp. Microtubules lacked electron dense granules. Dense granules, however were present in the distal portion of vp, juxtaposed with host myocyte. The ground sustance layer (gs) was up to $2.5 \mu \mathrm{m}$ thick and was relatively homogenous, except for a few dense granules. In tangentially cut sacrocysts, the gs sections of electron dense mt were scattered throughout the gs.

All sarcocysts contained few metrocytes and numerous mature bradyzoites. Both metrocytes and bradyzoites were arranged in groups (Fig. 2C). The bradyzoites were 11-15 $\mu \mathrm{m}$ long and 2.5-3.0 $\mu \mathrm{m}$ wide. They had the same structures as seen in other Sarcocystis bradyzoites, including micronemes, 2 rhoptries, a micropre, several dense granules, a nucleus, a mitochondrion, and numerous amylopectin granules (Fig. 4). The micronemes were in the conoidal third of the bradyzoite. Most micronemes were arranged in rows and were approximately $300 \times 50 \mathrm{~nm}$ in size with tapering or round ends (Fig.4).. The micropore was located $2.5 \mu \mathrm{m}$ from the tip of conoid. Granular material was seen below the micropore (Fig.4C).

\section{Discussion}

As stated earlier, there is considerable confusion concerning the species of Sarcocystis in equids. Rommel and Geisel (1975) in reviewing the earlier literature stated that Siedamgrotzky (1872) first reported sarcocysts in horses in Germany (we do not have access to this paper). He noticed cillia-like $2 \mu \mathrm{m}$ long protrusions on the cyst wall. Doflein (1901) named the parasite, 
Sarcocystis bertrami; he noticed cone-like structures on the cyst wall. Rommel and Geisel (1975) found thin-walled sarcocysts that lacked protrusions; they named the parasite Sarcocystis equicanis following the nomenclature proposed by Heydorn et al. (1975), combining the names of intermediate and definitive hosts. Gobel and Rommel (1980) described the ultrastructure of $S$. equicanis and noticed hair like 2-3 $\mu \mathrm{m}$ long protrusions on the cyst wall. Odening et al. (1995) expanded on the ultrastructure of sarcocysts in wild Equidae, and concluded that the structure broadly resembled the description of $S$. equicanis from the domestic horse.

In the present study, only one morphologic sarcocyst was found in the donkey and it is similar to the description of $S$. bertrami in the horse. Dubey et al. (2015) synonmyzed $S$. equicanis with $S$. bertrami. Illustrations in Figure 1 in the present study explain why the confusion arose concerning the thin-walled S. equicanis described by Rommel and Geisel (1875) and the hairy to thick walled sarcocysts described by Doflein (1901). In the present study both smooth and hairy portions were seen in the same cyst.

Gadaev (1978) found sarcocysts in 8 of 20 donkeys from the former USSR. He named the parasite S. asinus, solely based on the size, shape and staining of bradyzoites in smears. He said that brdyzoites of $S$. asinus were 1-3 $\mu$ m larger (12.3-17.5x 4.5-6.2 for $S$. asinus, and 11.514.5x 3.5-4.0 for S. bertrami) than S. bertrami of horses, and the nuclear chromatin was pale red in S. asinus versus crimson in S. bertrami (Gadaev, 1978). These differences are untenable for separating Sarcocystis species. Babudieri (1932) mentioned S. bertrami in a donkey in Italy but did not provide any details.

Hilali and Nassar (1987) found sarcocysts in 18 of 20 donkeys in Egypt. In histologic sections, sarcocysts were microscopic, up to $410 \mu \mathrm{m}$ long and $50.2 \mu \mathrm{m}$ wide. The sarcocyst wall was striated, and had villar protrusions that were up to $3.7 \mu \mathrm{m}$ long and up to $1 \mu \mathrm{m}$ wide (Hilali 
and Nassar, 1987). The bradyzoites were 16.2-16.9 x 4.1-4.2 $\mu \mathrm{m}$ in size. This description is generally similar to the present study.

Hinaidy and Loupal (1982) found sarcocysts in 1 of 2 donkeys in Austria and said that sarcocysts were the same as in the horse. They mentioned that sarcocysts in the donkeys were 1.7 to $14.1 \mathrm{~mm}$ long (Hinaidy and Loupal, 1982). However, they did not provide any detail and remarked that the cysts in the donkey were similar to those in the horse. All sarcocysts in the donkey in the present study were microscopic, and macroscopic sarcocysts were never found in donkeys in Egypt (Hilali, personal observations).

The description of the $S$. bertarami sarcocysts described here from the donkey should assist in the recognition of this parasite in horses and other equids.

\section{Acknowledgements}

We thank Dr. Petras Prakas for translation of the Gadaev paper.

\section{REFERENCES}

Babudieri, B. (1932). I sarcosporidi e le sarcosporidiosi. Archieves fur Protistenkunde. 76, 423-580.

Doflein, F. (1901). Sarcocystis bertrami n. sp. In: Die Protozoen als Parasiten und Krankheitserreger,nach biologischen Gesichtspunkten dargestellt. Gustav Fischer., Jena, pp. 219-220.

Dubey, J. P., Calero-Bernal, R., Rosenthal, B. M., Speer, C. A., and Fayer, R. (2015). Sarcocystosis of Animals and Humans. $2^{\text {nd }}$ edition. CRC Press. Boca Raton. FL, USA. 1-502.

Edwards, G.T. (1984). Prevalence of equine Sarcocystis in British horses and a comparison of two detection methods. Vet. Rec. 115, 265-267.

Erber, M., Geisel, O. (1981). Vorkommen und Entwicklung von 2 Sarkosporidienarten des Pferdes. Zeitschfrift fur Parasitenkunde (now Parasitology Research). 65, 283-291.

Gadaev, A. (1978). On sarcocysts of ass (Equus asinus). Akademii Nauk Uzbekskoi SSR 1, 47-48. (In Russian). 
Göbel, E. and Rommel, M. (1980). Licht- und elektronenmikroskopische Untersuchungen an Zysten von Sarcocystis equicanis in der Ösophagusmuskulatur von Pferden. Berliner und Münchener Tierärztliche Wochenschrift. 93, 41-47.

Heydorn, A. O., Gestrich, R., Mehlhorn, H. and Rommel, M. (1975). Proposal for a new nomenclature of the Sarcosporidia. Zeitschrift für Parasitenkunde. (Now Parasitology Research) 48, 73-82.

Hilali, M. and Nasser, A. M. (1987). Ultrastructure of Sarcocystis spp. from donkeys (Equus asinus) in Egypt. Veterinary Parasitology. 23, 179-183.

Hinaidy, H. K. and Loupal, G. (1982). Sarcocystis bertrami Doflein, 1901, ein Sarkosporid des Pferdes, Equus caballus. Zentralblatt fur Veterinarmedizin Reihe B. 29, 681-701.

Kirmse, P. (1986). Sarcosporidioses in equines of Morocco. British Veterinary Journal. 142, 70-72.

Levine, N.D., Tadros, W. (1980). Named species and hosts of Sarcocystis (Protozoa: Apicomplexa: Sarcocystidae). Systematic Parasitology. 2, 41-59.

Odening, K. (1998). The present state of species-systematics in Sarcocystis Lankester, 1882 (Protista, Sporozoa, Coccidia). Systematic Parasitology. 41, 209-233.

Odening, K., Wesemeier, H. H., Walter, G., and Bockhardt, I. (1995). Ultrastructure of sarcocysts from equids. Acta Parasitologica. 40, 12-20.

Rommel, M. and Geisel, O. (1975). Untersuchungen über die Verbreitung und de Lebenszyklus einer Sarkosporidienart des Pferdes (Sarcocystis equicanis n. spec.). Berliner und Münchener Tierärztliche Wochenschrift. 88, 468-471.

Van den Berg Weermans, M. A. and Dingemans, K. P. (1984). Rapid deparaffinization for electron microscopy. Ultrastructural Pathology. 7, 55-57.

Zayed, A.A., El-Ghaysh, A. (1998). Pig, donkey and buffalo meat as a source of some coccidian parasites infecting dogs. Veterinay Parasitology. 78, 161-168. 


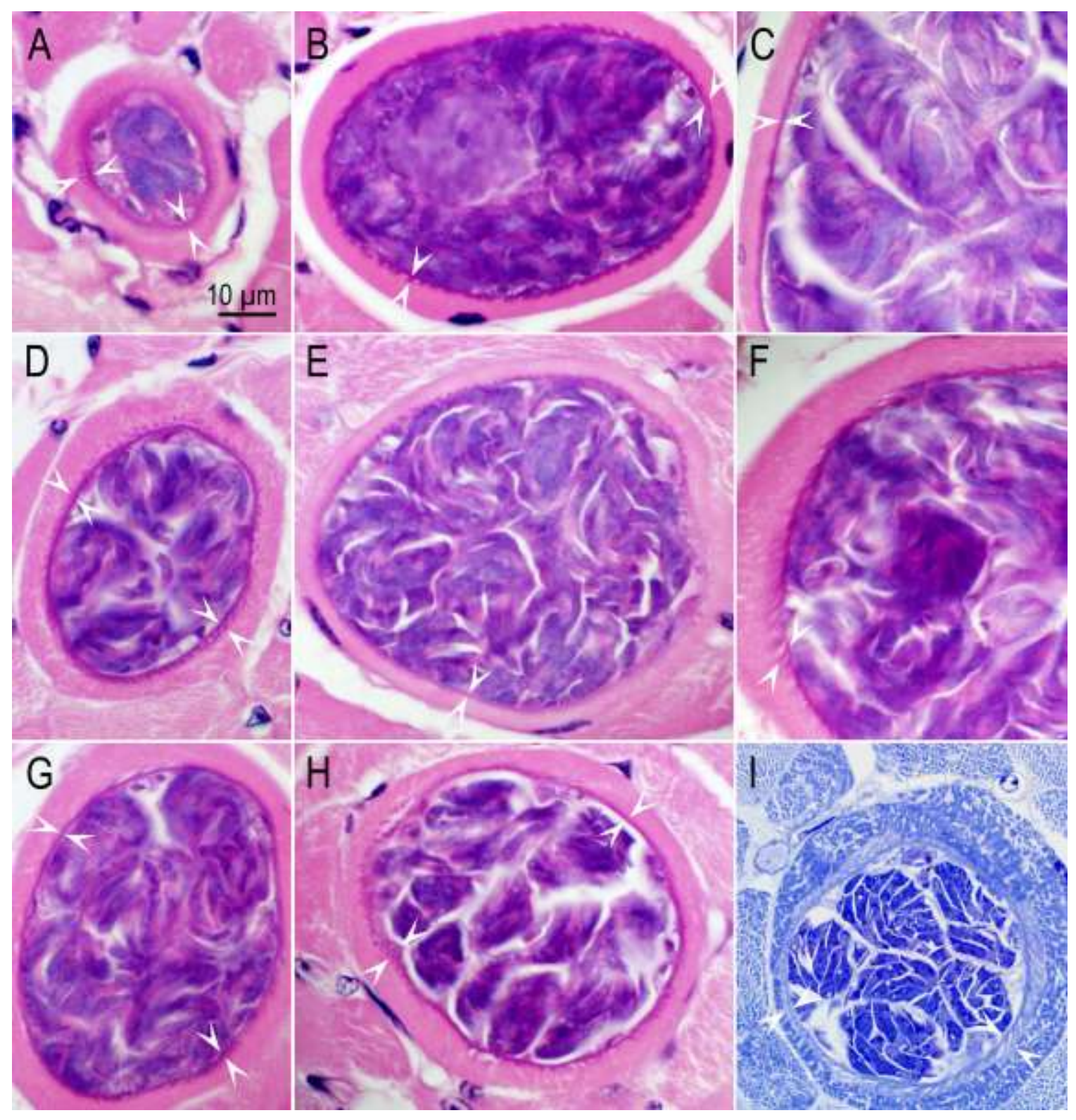

Fig. 1. Sarcocysts in histological sections of esophageal muscle of a donkey in Egypt. A-H, hematoxylin and eosin stain, I, Toluidine blues tain. Bar applies to all images. The sarcocyst wall appears smooth, striated, or cilliated (opposing arrowheads). 


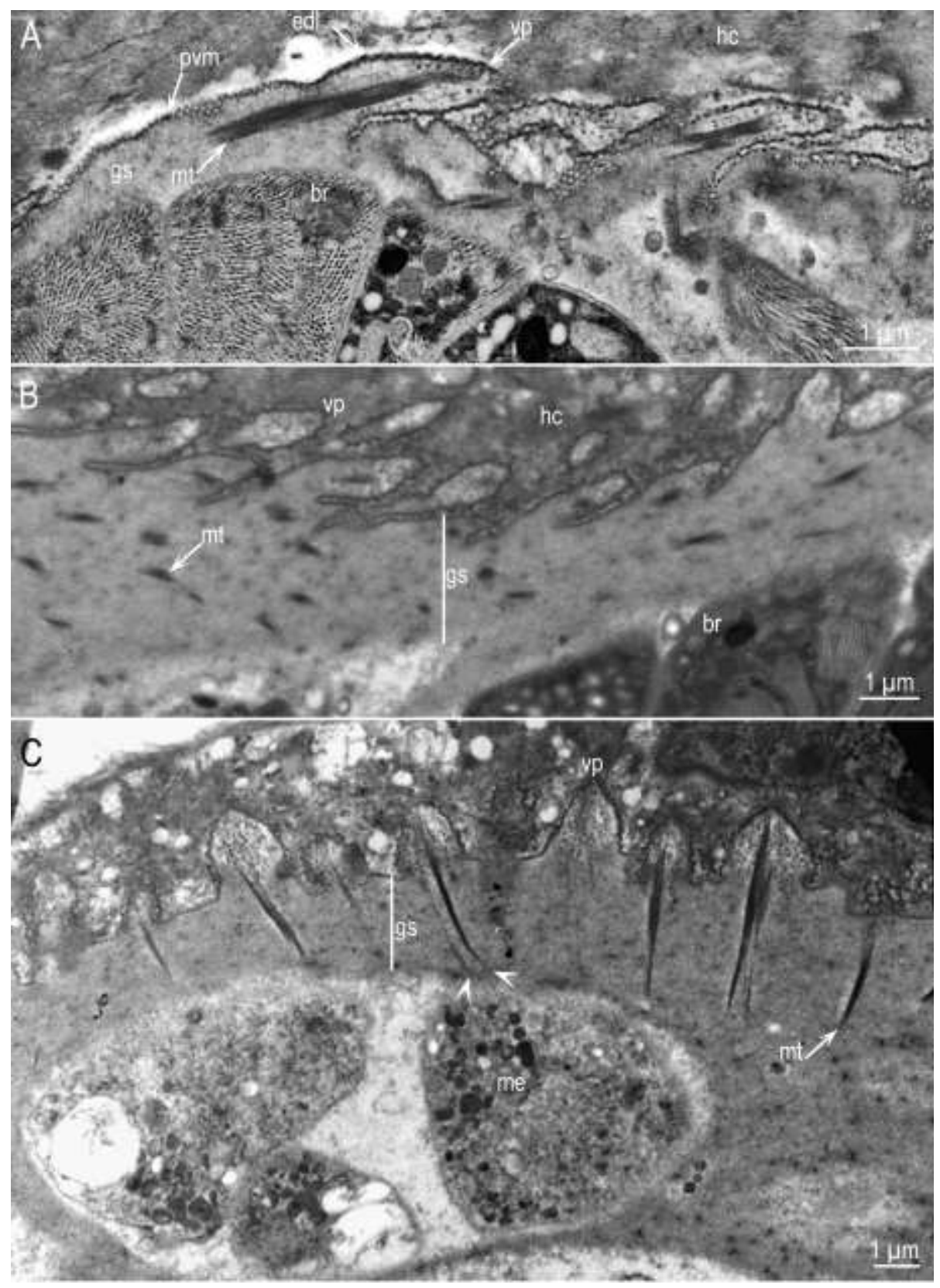

Fig. 2. TEM of $S$. bertami sarcocyst walls from 2 cysts. Glutarladehyde-fixed. Note parasitophorous vacuolar membrane (pvm) lined by electron dense layer, villar protrusions (vp), ground substance layers (gs), microtubules (mt), bradyzoites (br), metrocytes (me) and host cell (hc). (A) The vp are folded on the cyst wall. The gs is thin. Cyst \#1. (B) The vp are angled or folded over the cyst wall. The gs appears thick and has portions of microtubules (mt). (C). Same cyst as in B but the vp are conical, the gs is thick. The mt extend from the tip of the vp to the pellicle of metrocytes (arrowheads). Cyst \#2. 

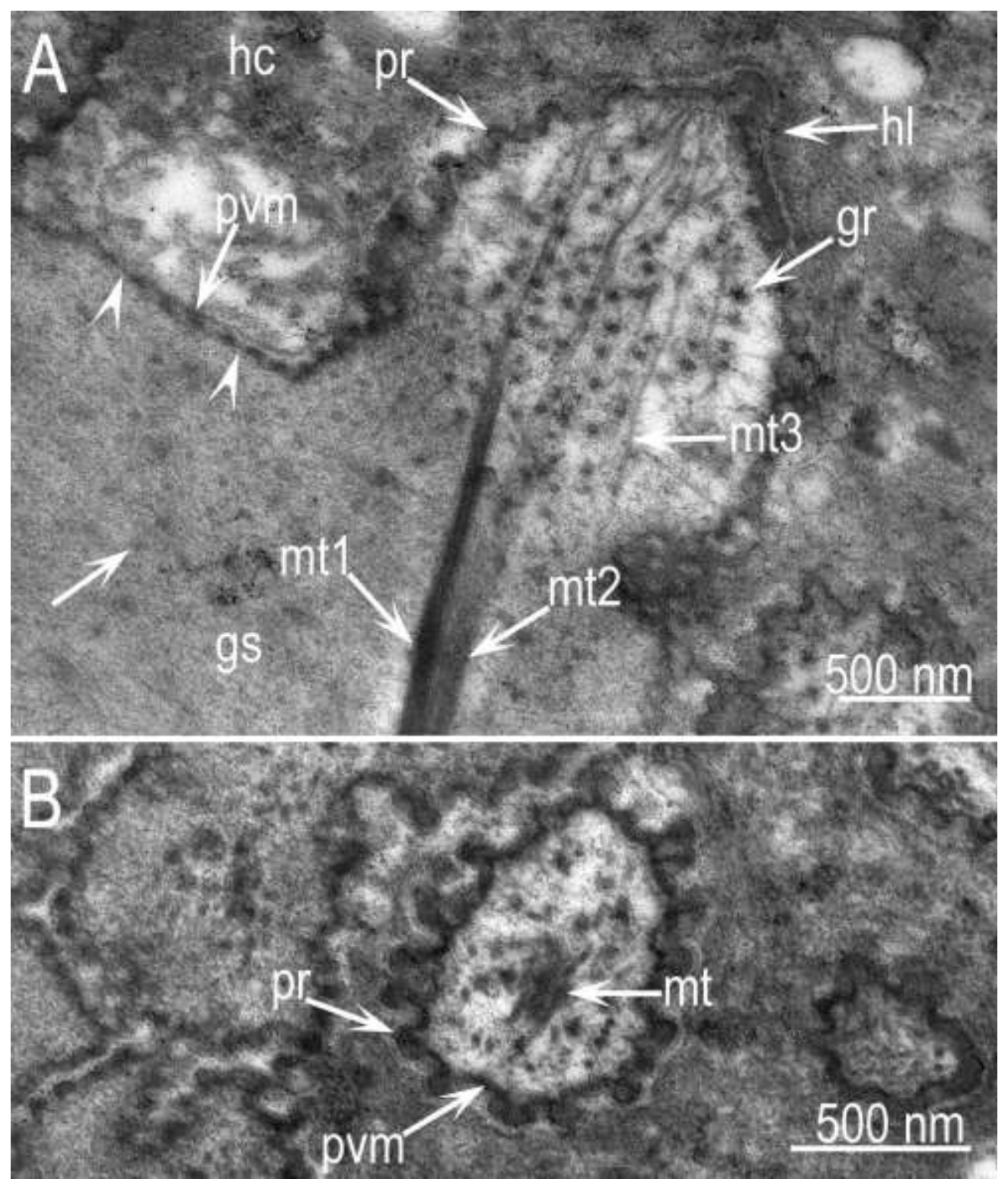

Fig. 3. TEM of $S$. bertami sarcocyst walls from cyst \#2. (A) Tip of a vp showing undulating pvm, lined by electron dense layer (edl), that is thinned out or absent at places (arrowheads). The pvm has outpocketing of protrusions (pr). Three types of microtubules are visible: (i) a thick tuft of electron dense microtubules (mt1), (ii) another tuft of medium electron dense microtubules (mt2), and (iii) fine mt that appear to criss cross towards the villar tips (mt3). Note numerous dense granules (gr) in the villar tips. A collar of hair-like (arrow) projections is present on vp. The gs is relatively smooth with few granules. (B) Cross section of vp showing protrusions (pr) on the pvm and mt. 
Fig. 4.TEM of S. bertami bradyzoites. Glutarladehyde-fixed. Note conoid (co), numerous micronemes (mn), several dense granules (dg), a nucleus (nu) and rhoptries (rh) with long slender neck. (A) A longitudinally cut bradyzoite. (B) Longitudinal section of conoidal part of a bradyzoite . Note conoid (co) with two rhoptries (rh1, rh2) with slender neck. The micronemes (mn) are arranged in rows. (C) Note a micropore (mp) with a collared rim (rm), dense granules (dg), and electron dense secretory material surrounds below the mp. 